\title{
Editorial
}

Herz 2015 • 40:835-836

DOI 10.1007/s00059-015-4348-6

Online publiziert: 22. August 2015

(c) Urban \& Vogel 2015

\section{R. Erbel}

Institut für Medizinische Informatik, Biometrie und Epidemiologie, Universitätsklinikum

Essen, Universität Duisburg-Essen, Essen, Deutschland

\section{Progression der koronaren Atherosklerose}

molekularbiologischer Sicht. Eindrucksvolle Bilder ergänzen ihre Aussagen.

Aus der sehr aktiven Arbeitsgruppe von Frau E. Regar stammt die Arbeit zur Demonstration der koronaren Neoatherosklerose mittels optischer Kohärenztomographie (OCT), die im Vergleich zum intravaskulären Ultraschall eine weiterführende Differenzierung der PlaqueMorphologie, vor allen Dingen im Bereich der Intima, und der Oberflächenstruktur ermöglicht.

Die Einführung des intravaskulären Ultraschalls vor 25 Jahren hat letztlich zur Akzeptanz der Methode geführt, um die Wirkung von Medikamenten zu testen. Der Surrogatparameter steht schneller zur Verfügung als langwierige Verlaufsuntersuchungen an großen Patientenkollektiven. Überzeugend konnten mehrere Studien, wie die ASTEROID- und SATURN-Studie, belegen, dass eine Regression atherosklerotischer nichtverkalkter Plaques möglich ist, wenn ein LDL-Wert unter den Schwellenwert von 75-80 mg/ dl abfällt. Die neuen Erkenntnisse werden vorgestellt.

Die Computertomographie (CT) eröffnet dem Arzt bei einem individuellen Patienten erstmalig die Möglichkeit, nicht-invasiv die koronare Atherosklerose $\mathrm{zu}$ diagnostizieren und anhand der Verkalkungsgrade zu differenzieren. A.A. Mahabadi und Mitarbeiter stellen die Methode im Rahmen der geführten Diskussion vor. Mittels Kontrastinjektion wird die CT zur Methode, die nicht nur verkalkte, sondern auch nicht verkalkte Plaques der Koronargefäße erfassen kann. P. Schoenhagen und F. Yuan aus Cleveland demonstrieren Daten zur Progression der koronaren Atherosklerose, erfasst mittels der CT -Angiographie.
Lange Zeit blieb die Koronarangiographie die einzige Möglichkeit, um die Progression und die Regression der koronaren Atherosklerose bei unseren Patienten zu demonstrieren. In einer Art Rückschau wird die Datenlage diesbezüglich vorgestellt und auf die interessante Analyse der SATURN-Studie mittels quantitativer Koronarangiographie eingegangen.

Die Herausgeber hoffen, dass Ihnen die Analysen der verschiedenen Arbeitsgruppen gefallen und Sie einen tieferen Einblick in die Bedeutung der Progression der koronaren Atherosklerose für die koronare Herzerkrankung gewinnen, die jetzt erstmals auch mit der CT nicht-invasiv erfasst und quantifiziert werden kann.

Prof. Dr. Raimund Erbel

\section{Korrespondenzadresse}

Prof. Dr. med. R. Erbel FAHA, FESC, FASE, FACC

Institut für Medizinische Informatik, Biometrie und Epidemiologie

Universitätsklinikum Essen

Universität Duisburg-Essen

Hufelandstr 55, 45147 Essen

erbel@uk-essen.de

\section{Literatur}

1. Lichtlen PR, Hugenholtz PG, Rafflenbeul W, Hecker H, Jost S, Deckers JW (1990) Retardation of angiographic progression of coronary artery disease by nifedipine. Results of the International Nifedipine Trial on Antiatherosclerotic Therapy (INTACT). INTACT Group Investigators. Lancet 335:1109-1113

2. Brown G, Albers JJ, Fisher LD, Schaefer SM, Lin JT, Kaplan C, Zhao XQ, Bisson BD, Fitzpatrick VF, Dodge HT (1990) Regression of coronary artery disease as a result of intensive lipid-lowering therapy in men with high levels of apolipoprotein B. N Engl J Med 323:1289-1298 der Arbeitsgruppe von H. Baba aus Essen beschreiben zunächst die Atherosklerose der Koronararterien und die Plaque-Progression aus histomorphologischer und 
3. Ballantyne CM, Raichlen JS, Nicholls SJ, Erbel R, Tardif JC, Brener SJ, Cain VA, Nissen SE; ASTEROID Investigators (2008) Effect of rosuvastatin therapy on coronary artery stenoses assessed by quantitative coronary angiography: a study to evaluate the effect of rosuvastatin on intravascular ultrasoundderived coronary atheroma burden. Circulation 117:2458-2466

4. von Birgelen C, Hartmann M, Mintz GS, Baumgart D, Schmermund A, Erbel R (2003) Relation between progression and regression of atherosclerotic left main coronary artery disease and serum cholesterol levels as assessed with serial long-term (> or $=12$ months) follow-up intravascular ultrasound. Circulation 108:2757-2762

5. von Birgelen C, Hartmann M, Mintz GS, van Houwelingen KG, Deppermann N, Schmermund A, Böse D, Eggebrecht H, Neumann T, Gössl M, Wieneke $H$, Erbel $R$ (2004) Relationship between cardiovascular risk as predicted by established risk scores versus plaque progression as measured by serial intravascular ultrasound in left main coronary arteries. Circulation 110:1579-1585

6. Nissen SE, Nicholls SJ, Sipahi I, Libby P, Raichlen JS, Ballantyne CM, Davignon J, Erbel R, Fruchart JC, Tardif JC, Schoenhagen P, Crowe T, Cain V, Wolski K, Goormastic M, Tuzcu EM; ASTEROID Investigators (2006) Effect of very high-intensity statin therapy on regression of coronary atherosclerosis: the ASTEROID trial. JAMA 295:1556-1565

7. Nissen SE, Tuzcu EM, Schoenhagen P, Brown BG, Ganz P, Vogel RA, Crowe T, Howard G, Cooper CJ, Brodie B, Grines CL, DeMaria AN; REVERSAL Investigators (2004) Effect of intensive compared with moderate lipid-lowering therapy on progression of coronary atherosclerosis: a randomized controlled trial. JAMA 291:1071-1080

8. Nicholls SJ, Borgman M, Nissen SE, Raichlen JS, Ballantyne C, Barter P, Chapman MJ, Erbel R, Libby $P$ (2011) Impact of statins on progression of atherosclerosis: rationale and design of SATURN (Study of Coronary Atheroma by InTravascular Ultrasound: effect of Rosuvastatin versus AtorvastatiN). Curr Med Res Opin 27:1119-1129

9. Nicholls SJ, Ballantyne CM, Barter PJ, Chapman MJ, Erbel RM, Libby P, Raichlen JS, Uno K, Borgman M, Wolski K, Nissen SE (2011) Effect of two intensive statin regimens on progression of coronary disease. N Engl J Med 365:2078-2087

\section{Bei Herzüberlastung: Stetiger Einstrom von Kalzium schwächt das Herz}

Kalzium-leitende Proteine spielen entscheidende Rolle bei krankhaftem Herzmuskelwachstum. Inaktivierung dieser Proteine schützt im Tierversuch vor Herzschäden

Wissenschaftler des Instituts für Pharmakologie am Universitätsklinikum Heidelberg haben in Zusammenarbeit mit Zellbiologen der Universität des Saarlandes einen entscheidenden Mechanismus bei der Entstehung chronischer Herzschwäche entdeckt: Ist das Herz dauerhafter Überlastung z.B. durch Bluthochdruck ausgesetzt, lassen zwei bestimmte Proteine von lonen-Kanälen unablässig Kalzium in die Muskelzellen einströmen. Dies setzt krankhafte Umbauprozesse des Herzmuskels in Gang. Werden die Proteine im Tierversuch ausgeschaltet, bleibt das Herz - ohne dass Schlagkraft und Herzrhythmus darunter leiden - trotz gleicher Belastung leistungsfähig und die Veränderungen fallen nur gering aus. Der Kalzium-Signalweg könnte daher einen lohnenden Ansatzpunkt für neue Therapien zur gezielten Vorbeugung der chronischen Herzschwäche bieten.

Dauerhaft erhöhter Blutdruck, Erkrankungen der Herzklappen sowie Engstellen an der Aorta bedeuten Schwerstarbeit für das Herz. Es kompensiert diese Belastung durch Hypertrophie und Fibrosierung. Chronische Herzschwäche, Rhythmus $\neg$ störungen oder plötzlicher Herztod können die Folgen sein. Weitere Risikofaktoren sind Übergewicht und Alter: Mehr als 40 Prozent der über 70-Jährigen leiden an einer Herzmuskel $\neg$ hypertrophie. Bisher gibt es keine Therapie, die diesen Krankheitsmechanismus, das maladaptive kardiale Remodeling, auf molekularer Ebene stoppt.

Die Wissenschaftler untersuchten in wie weit ein wichtiger Signalgeber des Herzens, Kalzium, in diesen Mechanismus involviert ist oder diesen sogar in Gang setzten kann. Es gab Hinweise, dass Kalzium auch eine Rolle bei der Anpassung der Herzmuskelzellen an Belastung spielt - und zwar unabhängig von seiner Funktion bei Herzschlag und Herzrhythmus. Dazu benötigt es separate KalziumKanäle, die unabhängig vom Herzschlag funktionieren. Zwei entscheidende Kanalproteine, TRPC1 und TRPC4, wurden nun entdeckt.
TRPC1 und TRPC4 gehören einer Gruppe ähnlicher Proteine an, die sich durch die Hormone Adrenalin und Angiotensin II, die beide den Blutdruck erhöhen und auch direkt auf den Herzmuskel wirken, aktivieren lassen. Die Wissenschaftler überprüften die Funktion dieser Proteine mit Hilfe genetisch veränderter Mäuse, die jeweils einzelne oder mehrere von insgesamt sechs TRPC-Proteinen nicht bilden konnten. Sie zeigten: Unter der Wirkung von Angiotensin II und Adrenalin kommt es zu einer Steigerung eines kontinuierlichen Einstroms von Kalzium in die Herzzellen. Bei Herzzellen von Mäusen, denen Protein TRPC1 und gleichzeitig TRPC4 fehlten, war dieser Kalziumeinstrom und damit auch der Kalziumspiegel in den Zellen dauerhaft deutlich vermindert. Im lebenden Tier fiel ohne diese beiden Eiweiße trotz künstlich herbeigeführtem Bluthochdruck oder Aortenverengung das krankhafte Muskelwachstum des Herzens nur gering aus. Die Mäuse litten auch unter deutlich weniger Herzfunktionsstörungen als normale Mäuse unter chronischer Druckbelastung. Die Tiere ohne TRPC1 und TRPC4 waren über die Versuchsdauer vor einer Herzschwäche geschützt. Bei Tieren, denen jeweils nur TRPC1 oder nur TRPC4 fehlte, waren diese Effekte nicht zu beobachten.

Durch das Fehlen der beiden Kanalproteine traten keine Nebenwirkungen auf, wie dies häufig der Fall ist, wenn einzelne Signalwege im Herzen blockiert werden: Unter normalen Bedingungen zeigen die genetisch veränderten Mäuse keine Auffälligkeiten; ihre Herzen funktionierten und reagierten normal. Ein nächster Schritt ist es nun, entsprechende Wirkstoffe zu entwickeln und zu testen, die ausschließlich TRPC1 und TRPC4 blockieren.

Literatur:

Camacho Londoño J. E., Tian Q, Hammer K et al (2015) A background Ca21 entry pathway mediated by TRPC1/TRPC4 is critical for development of pathological cardiac remodelling European Heart Journal, doi:10.1093/ eurheartj/ehv250 